\title{
Association between diet quality scores, adiposity, glycemic status and nutritional biomarkers among Indian population with type 2 diabetes mellitus: A cross-sectional study
}

\author{
Aamir Bashir ${ }^{\mathrm{a}}$, Krishna Pandey ${ }^{\mathrm{b}, * *}$, Md Azharuddin $^{\mathrm{c}}$, Anjali Kumari ${ }^{\mathrm{d}}$, Ishfaq Rashid ${ }^{\mathrm{e}}$, \\ N.A. Siddiqui ${ }^{\mathrm{f}}$, Chandra Shekhar Lal $^{\mathrm{c}}$, Krishna Murti ${ }^{\mathrm{a}, *}$ \\ ${ }^{\text {a }}$ Department of Pharmacy Practice, National Institute of Pharmaceutical Education and Research, Hajipur, Bihar, 844102, India \\ ${ }^{\mathrm{b}}$ Division of Clinical Medicine, Rajendra Memorial Research Institute of Medical Sciences (Indian Council of Medical Research), Agamkuan, Patna, 800007, Bihar, India \\ ${ }^{\mathrm{c}}$ Department of Pharmaceutical Medicine (Division of Pharmacology), School of Pharmaceutical Education and Research, Jamia Hamdard, New Delhi, 110062, India \\ ${ }^{\mathrm{d}}$ Division of Biochemistry, Rajendra Memorial Research Institute of Medical Sciences (Indian Council of Medical Research), Agamkuan, Patna, 800007, Bihar, India \\ ${ }^{\mathrm{e}}$ Department of Pharmacy Practice, National Institute of Pharmaceutical Education and Research, SAS Nagar, Punjab, 160062, India \\ ${ }^{\mathrm{f}}$ Division of Biostatistics, Rajendra Memorial Research Institute of Medical Sciences (Indian Council of Medical Research), Agamkuan, Patna, 800007, Bihar, India
}

\section{A R T I C L E I N F O}

\section{Keywords:}

Dietary pattern

Nutritional biomarkers

Dietary score

Type 2 diabetes mellitus

Cardiovascular diseases

\begin{abstract}
A B S T R A C T
Background: Unhealthy dietary scores have been associated with increased risk of Type 2 Diabetes mellitus, which have a significant impact on affected individuals and healthcare services. However, there is lack of published evidence on quality of diet in type 2 diabetic subjects. Therefore, we aimed to assess dietary pattern scores and their relationship with adiposity, glycemic profile, lipid parameters and nutritional biomarkers in T2DM patients.

Methods: We included T2DM patients $(n=203)$ with age group ranging from 30-70 years. All patients were interviewed for their dietary patterns through a derivative questionnaire of UK Diabetes and Diet Questionnaire (UKDDQ). Patient information including socio-demographic and anthropometric details were obtained. All biochemical parameters were estimated and recorded. The SPSS ver. 21 was used to perform statistical analysis. Results: The study included $48.8 \%$ males and $51.2 \%$ females with mean age of $53.92 \pm 10.56$ years. The mean values of diet scores ranged from healthiest $[1.02 \pm 0.17(\mathrm{CI}=1.00$ to 1.04$)]$ to least healthy $[2.65 \pm 0.71(\mathrm{CI}=2.55$ to 2.74$)]$ respectively. Majority of patients $(\mathrm{n}=153)$ were reported to have a habit of skipping breakfast routinely within $2 \mathrm{~h}$ after waking. However, 162 patients were consuming the unnecessary food items between the regular meals. While increasing in diet mean scores i.e. moving from lower quintile Q1(21.86 \pm 1.23$)$ to higher quintile Q5(28.94 \pm 2.24$)$, a variable relationship was found. The post prandial (PP2) $(r=0.233)$, fasting blood glucose $(F B G)(r=0.183), \operatorname{HbA1c}(r=0.253)$ and body mass index (BMI) $(r=0.139)$ shown a direct association whereas $\operatorname{zinc}(r=-0.116)$, vitamin $\mathrm{D}(\mathrm{r}=-0.185)$ and Albumin $(\mathrm{r}=-0.043)$ were inversely correlated between the quintiles.

Conclusions: Increasing dietary scores are supposed to have a provoking effect on disease condition resulting into worse outcomes at higher scores. The findings from nutritional biomarkers suggested that deficiently found nutrients must be supplemented as a part of therapy to enhance the treatment benefits.
\end{abstract}

\section{Introduction}

Diabetes mellitus is a metabolic-cum vascular chronic disorder resulting from impaired insulin physiology. ${ }^{1}$ It causes chronic hyperglycemia leading to different progressive and life-threatening complications like cardiovascular disease (CVD), neuropathy, nephropathy, retinopathy and other organ damages. ${ }^{2}$ Type 2 diabetes mellitus (T2DM), considered as a pandemic of this century is the most common form of Diabetes mellitus contributing approximately $90 \%$ of all diabetic cases. ${ }^{3,4}$ The global diabetic prevalence is $8.8 \%$ and India is accounting 72.9 million diabetics, whereas 42.2 million undiagnosed diabetics estimated with a prevalence of $8.5 \%$ in South-East Asia. ${ }^{4}$

\footnotetext{
* Corresponding author.

** Corresponding author.

E-mail addresses: drkrishnapandey@yahoo.com (K. Pandey), krishnamurti74@gmail.com (K. Murti).
} 
Recently, a study reported the prevalence of T2DM is higher, approximately $10 \%$, in the states with greater per-capita income like Punjab and lower $4.3 \%$, in low per-capita income state like Bihar. ${ }^{5}$

Dietary patterns are described as the overall diet consisting of various combinations of foods and nutrients consumed by an individual. Studies on dietary patterns have been adopted recently in nutritional epidemiology which incorporates the cumulative influence of composite diet rather than individual food components or nutrients, as in the conventional approach, on health outcomes. ${ }^{6}$

The dietary patterns with their nutritional status play a critical role in the pathogenesis of T2DM along with other modifiable risk factors like obesity, physical inactivity, sedentary lifestyles and pre-diabetes state $^{7,8}$ Evidence from clinical trials and observational studies have suggested that the consumption of different food patterns and beverages are associated with a reduced risk of diabetes. These patterns include a high intake of whole grains, vegetables, nuts, coffee, and a moderate consumption of alcohol and low intake of sugar-sweetened drinks sugary-sweetened beverages, processed and unprocessed red meats. $^{9-11}$

For improving the food habit of any population effectively, it is necessary to understand what individuals are eating and what key nutrients are being supplied by their dietary behaviors. ${ }^{12,13}$ Dietary patterns need to be assessed for some important nutritional biomarkers like cholesterol, zinc, vitamin D, calcium, albumin as these biomarkers play an important role in T2DM pathophysiology. ${ }^{14-16}$ Thus their association with onset and progression of diabetes must be identified. The association of dietary pattern with consideration of these essential nutrients for T2DM is a better measure than evaluating the relationship between individual nutrients and disease separately as people are not aware of concept of specific nutrients individually. ${ }^{17}$ Currently, it is an emerging area of research to interpret the dietary patterns through different assessment tools which can be used by healthcare professionals or researchers to guide diabetic patients for their dietary modifications. ${ }^{18}$ Dietary patterns in India are highly diverse and heterogeneous, thus the process of food pattern evaluation is very complex. In addition, a study shown that individual nutrient intakes are being focused instead of assessing diet as a whole in South Asian populations. ${ }^{19}$ However, there is lack of evidence on association between dietary patterns and nutrients with health outcomes for Indian diabetic patients. $^{20}$

Therefore, we aimed to explore the association between dietary pattern scores with cardio-metabolic risk factors and status of nutritional biomarkers in patients with Type 2 diabetes mellitus in the population of Bihar, an eastern state of India.

\section{Material and methods}

\subsection{Study design}

This prospective observational study was conducted at the outpatient department (OPD) of Rajendra Memorial Research Institute of Medical Sciences-Indian Council of Medical Research (RMRIMS-ICMR) Patna, Bihar. The study protocol was approved by the Institutional Ethics Committee of Rajendra Memorial Research Institute of Medical Sciences (RMRIMS), Patna. Written informed consent was obtained from all study participants and was assured of their confidentiality and anonymity. ${ }^{21}$ The manuscript was written in accordance with the STROBE guidelines. ${ }^{22}$

\subsection{Sample size calculation}

A simple random sampling technique was utilized for the selection of study subjects. The sample size calculation was attempted for quantitative variable using standardized statistical formula. ${ }^{23}$ The sample size was calculated based upon an assumed prevalence of $8.5 \%$ in a general hospital setting of Bihar for T2DM cases, with a precision of
$5 \%$ at $95 \%$ confidence level and was found to be 117 (diabetic adults aged between 30 and 70). Taking into consideration of $10 \%$ non-response, the final sample size was rounded to 150 .

\subsection{Subject selection}

In this study, 233 type 2 diabetic patients were enrolled. Among them, 30 patients were excluded with the reason; unwilling to participate $(\mathrm{n}=17)$ and patients dropped out prior to completing the information $(n=13)$. Finally, 203 patients met the inclusion criteria for the quantitative analysis including 99 male subjects and 104 females. Participants were eligible to take part in this study if the age group ranges between 30 and 70 years, coming from different regions of Bihar. The patients were included in this study, who had been diagnosed with type 2 diabetes mellitus with fasting blood sugar (FBS) level $\geq 126 \mathrm{mg} / \mathrm{dl}$. Patients were taking anti-diabetic medication from more than 6 months. Exclusion criteria included; patients with the co-morbidities other than hypertension and dyslipidemia, not agree to sign the informed consent, presence of a condition likely to alter dietary requirements e.g., food allergy or intolerance, pregnancy or lactation.

\subsection{Data collection}

Data collection was done by using a derivative of UKDDQ version 2.0 (specific for South East Asians) in the general outpatient department (OPD) through direct interview of patients by an experienced interviewer. The questionnaire was used in English and also translated into Hindi, the local language spoken in the study region for the convenience of the participants. ${ }^{24}$

\subsubsection{Demographic data}

Socio-demographic details, such as name, gender, age, marital status, level of education, income, family history of diabetes, addictions, any diabetic complications, and other complications hypertension and dyslipidemia.

\subsubsection{Dietary assessment}

Assessment of dietary pattern was done by using a derivativequestionnaire of UK Diabetes and Diet Questionnaire (UKDDQ) which is supposed to be a brief, reliable and valid diet assessment tool. ${ }^{24}$ The derivative was developed specifically for this study to evaluate food habit by considering the nature of food group, the frequency of consumption, how much taking (illustrated by portion size photographs). ${ }^{25}$ Out of 20 items, 16 questions contributed for the total dietary score. Other 4 questions were for evaluating weight concern, level of physical exercise, diet change importance and confidence to change diet respectively.

Enrolled patients were given all detailed information for every dietary habit being assessed. They were asked to choose how frequently they are consuming the different food items from different dietary groups over the last one month. Interviewed subjects were given different options to choose ranging from 'Never, very rarely or less than once a week, '1-4 times a week, ' 5 times to every day in a week' and likewise for different food habits. The answers were noted by different letters as A's, B's and C's equivalent to healthy, moderately healthy and unhealthy dietary choices respectively. For further data interpretation, the letters were again coded into numerical values as $A=1, B=2$, $\mathrm{C}=3$ respectively. ${ }^{12}$ The possible range of scores for food habit questions opted by participants could be from 16 to 48 with an intention that lower scores reflect healthily, middle scores reflect moderately healthy and higher scores reflect unhealthy dietary choices respectively.

\subsubsection{Anthropometric, clinical and biochemical measurements}

Anthropometric measurements were obtained at the time of the interview using standardized procedures. Height was measured using a 
wall-mounted height measurement scale, to the nearest $0.5 \mathrm{~cm}$. A standard tape was used to measure Waist circumference (WC) nearest to inches in the horizontal plane just above the umbilicus. Body weight was measured to an accuracy of $0.1 \mathrm{~kg}$ and body mass index (BMI) computed as BMI = body mass $(\mathrm{kg}) /[$ height $(\mathrm{m})]{ }^{2}$

Venous blood ( $2 \mathrm{~mL}$ ) was collected after overnight fasting in serum and EDTA-Vacutainers (BD, USA) for biochemical measurements. American Diabetes Association(ADA) recommendations were followed to diagnose diabetes mellitus. Fasting blood glucose (FBG) level of $\geq 126 \mathrm{mg} / \mathrm{dL}$ after a minimum 12-h fasting and 2-h after meal glucose level (OGTT) of $\geq 200 \mathrm{mg} / \mathrm{dL}$ were considered to differentiate between diabetic and non-diabetic subjects. ${ }^{26}$ Blood glucose level was estimated by using the commercially available kit (Meril, India) following manufacturers' instruction. HbA1c and lipid profile were analyzed by using the AlereAfinion diagnostic kit with Afinion ${ }^{\mathrm{TM}}$ AS100 Analyser (Alere, USA). Vitamin D was estimated by 25-(OH) Vitamin D ELISA diagnostic kit (Calbiotech, USA) following instructions given in the instruction manual. Other parameters like urea (GLDH-method), creatinine (Jaff's kinetic method), zinc (Colorimetric method), calcium (Arsenazo III method) and albumin(BCG Dye method) were analyzed by using commercially available kits by Microlab 300 clinical chemistry analyser (Merck, Netherlands).

\subsubsection{Assessment of other covariates}

Covariates were analyzed at baseline of study for socio-demographic variables (age, sex, occupation, diet, religion, marital status, monthly income, educational qualification), anthropometric parameters (weight, height, BMI, W.C), lifestyle factors (addiction, physical exercise), family history of diabetes, duration of diabetes and other complications (hypertension or dyslipidemia). ${ }^{3}$ Occupation was categorized into 3 categories as not working, labourer/farmer and businessman/employee. Educational qualification was grouped into primary, matriculate, intermediate, graduate and postgraduate. Monthly income was assessed by 4 groups as, less than 5000, 5000-10000, 10000-20000 and more than 20000 [INR]. The complications were categorized into four groups as no complications, hypertension, dyslipidemia, and both hypertension and dyslipidemia. Physical exercise was evaluated by four categories as; as per recommended, moderately exercising, day to day activities and never exercising.

\subsubsection{Statistical analysis}

Data were processed in Excel-sheet and analyzed using the SPSS software (Version 21; CDC Atlanta, USA). Socio-demographic variables and dietary patterns were analyzed by using descriptive statistics. Frequencies and percentages were calculated for categorical variables whereas other continuous baseline data variables were shown as mean \pm Standard Deviation (SD). Dietary habits were presented by mean $\pm \mathrm{SD}$, confidence interval(CI), median and inter-quartile range (IQR). The total dietary score (mean $\pm \mathrm{SD}$ ) was divided into quintiles as $21.86 \pm 1.23$ for Q1, $24.84 \pm 0.76$ for Q3 and $28.94 \pm 2.24$ for Q5. The significant differences between the different anthropometric, biochemical and nutritional parameters were estimated by One way 'ANOVA' between the three quintiles. Furthermore, the Pearson's correlation analysis was performed in order to assess the association between dietary scores and said parameters for evaluating the significant associations. All tests were two-tailed and statistical significance was set at the probability value $(\mathrm{P}<0.05){ }^{27}$

\section{Results}

The socio-demographic characteristics of patients are shown in Table 1 . The mean age of the patients was $53.92 \pm 10.56$ years, ranged from 30 to 70 years. Among the participants, $57 \%$ patients were unemployed, 157 were having a Non-vegetarian dietary habit. The male patients $(n=50)$ were addicted to Tobacco chewing. Of participants, 89 were educated up to primary level and 97 patients were reported to
Table 1

Distribution based on socio-demographic details.

\begin{tabular}{|c|c|c|c|}
\hline Variables & & $(\mathrm{N}=203)$ & $\%$ \\
\hline \multirow[t]{2}{*}{ Gender } & Male & 99 & 48.8 \\
\hline & Female & 104 & 51.2 \\
\hline \multirow[t]{3}{*}{ Occupation } & Not working & 116 & 57.14 \\
\hline & Labourer/Farmers & 19 & 9.35 \\
\hline & Businessman/Employee & 68 & 31.52 \\
\hline \multirow[t]{2}{*}{ Diet } & Vegetarian & 46 & 22.66 \\
\hline & Non-vegetarian & 157 & 77.34 \\
\hline \multirow[t]{4}{*}{ Addiction } & No addiction & 145 & 71.42 \\
\hline & Tobacco chewing & 50 & 24.63 \\
\hline & Alcohol & 1 & 0.004 \\
\hline & Multiple & 7 & 0.03 \\
\hline \multirow[t]{5}{*}{ Education } & Primary & 89 & 43.84 \\
\hline & Matriculation & 59 & 29.06 \\
\hline & Intermediate & 18 & 8.86 \\
\hline & Graduation & 28 & 13.79 \\
\hline & Post-graduation & 9 & 4.43 \\
\hline \multirow[t]{4}{*}{ Income (Monthly In INR) } & $<5000$ & 97 & 47.78 \\
\hline & $5000-10000$ & 39 & 19.21 \\
\hline & $10000-20000$ & 29 & 14.28 \\
\hline & $>20000$ & 38 & 18.71 \\
\hline \multirow[t]{2}{*}{ Family History } & No history & 141 & 69.45 \\
\hline & Primary relatives & 62 & 30.54 \\
\hline \multirow[t]{4}{*}{ Other Complication } & No complication & 78 & 38.42 \\
\hline & Hypertension & 62 & 30.54 \\
\hline & Dislipidemia & 31 & 15.27 \\
\hline & Both HTN/Dislipidemia & 32 & 15.76 \\
\hline
\end{tabular}

Abbreviations: $\mathrm{INR}=$ Indian rupee.

have a monthly income of less than 5000 INR. The proportion of patients having a family history of diabetes and hypertension was found to be $30 \%$ and $15 \%$ had dyslipidemia whereas 32 patients showed both complications i.e. hypertension and dyslipidemia simultaneously. ${ }^{28}$

Clinical characteristics of included subjects are summarized in the Table 2. Females were significantly higher from males, w.r.t BMI, WC and WHtR $(\mathrm{p}<0.01)$. Mean WC exceeded the recommended cut-off $(>80 \mathrm{~cm})$ in both male and female groups indicating abdominal

Table 2

Profiling of study subjects based on clinical characteristics.

\begin{tabular}{llll}
\hline Variables & $\begin{array}{l}\text { Male Diabetic } \\
\text { Group(N }=99)\end{array}$ & $\begin{array}{l}\text { Female Diabetic } \\
\text { Group(N=104) }\end{array}$ & P-value \\
\hline Age & $55.63 \pm 10.15$ & $52.30 \pm 10.72$ & 0.025 \\
Weight $(\mathrm{Kg})$ & $63.75 \pm 9.76$ & $60.71 \pm 11.60$ & 0.046 \\
Height $(\mathrm{cm})$ & $158.27 \pm 7.87$ & $151.15 \pm 6.27$ & 0.000 \\
BMI $\left(\mathrm{Kg} / \mathrm{m}^{2}\right)$ & $25.27 \pm 3.02$ & $26.22 \pm 3.79$ & 0.052 \\
W.C $(\mathrm{cm})$ & $87.35 \pm 6.55$ & $90.42 \pm 7.49$ & 0.002 \\
WHtR & $0.55 \pm 0.83$ & $0.59 \pm 1.19$ & 0.01 \\
Systolic pressure & $133.05 \pm 13.26$ & $135.58 \pm 14.73$ & 0.201 \\
Diastolic pressure & $85.05 \pm 6.79$ & $86.80 \pm 6.69$ & 0.067 \\
FBG & $157.89 \pm 73.38$ & $178.01 \pm 74.61$ & 0.054 \\
PP2 & $241.60 \pm 104.49$ & $257.92 \pm 108.35$ & 0.276 \\
HbA1c & $8.08 \pm 2.22$ & $8.48 \pm 2.44$ & 0.221 \\
Triglyceride (TG) & $123.11 \pm 67.01$ & $153.39 \pm 78.16$ & 0.003 \\
Total cholesterol (TC) & $162.01 \pm 37.22$ & $177.49 \pm 40.361$ & 0.005 \\
LDL & $90.42 \pm 38.01$ & $100.15 \pm 34.44$ & 0.057 \\
HDL & $44.06 \pm 17.76$ & $46.41 \pm 12.43$ & 0.274 \\
Non-HDL & $113.98 \pm 32.97$ & $129.12 \pm 38.27$ & 0.003 \\
CHO/HDL-ratio & $3.85 \pm 1.08$ & $4.33 \pm 4.71$ & 0.325 \\
Urea & $36.97 \pm 22.22$ & $34.39 \pm 4.45$ & 0.248 \\
Creatinine & $1.07 \pm 1.09$ & $0.84 \pm 0.23$ & 0.039 \\
Zinc & $53.94 \pm 10.96$ & $54.42 \pm 10.50$ & 0.749 \\
Vitamin D & $26.12 \pm 7.16$ & $26.15 \pm 6.63$ & 0.974 \\
Albumin & $3.64 \pm 0.36$ & $3.65 \pm 0.45$ & 0.931 \\
Calcium & $9.50 \pm 1.55$ & $9.74 \pm 2.00$ & 0.330 \\
\hline Abbreviations: & & & \\
\hline
\end{tabular}

Abbreviations: $\quad \mathrm{BMI}=$ body mass index, $\quad$ W.C $=$ Waist circumference, $\mathrm{WHtR}=$ waist-to-height ratio, $\mathrm{FBG}=$ fasting blood glucose, $\mathrm{PP} 2=$ Post parandial: $2 \mathrm{~h}$ after meal glucose, HbA1c $=$ glycated haemoglobin, $\mathrm{HDL}=$ high density lipoprotein, $\mathrm{LDL}=$ low density lipoprotein, $\mathrm{CHO}=$ cholesterol. 
Table 3

Dietary assessment by utilizing the derivative of UK Diabetes and Diet Questionnaire (UKDDQ).

\begin{tabular}{|c|c|c|c|c|}
\hline Food Habit Assessment( $\mathrm{N}=203)$ & Mean \pm SD & $95 \% \mathrm{CI}$ & Median(IQR) & $\mathrm{N}(\%)$ patients with healthy scores \\
\hline Breakfast within $2 \mathrm{~h}$ after waking ${ }^{\mathrm{a}}$ & $2.43 \pm 0.86$ & 2.31 to 2.55 & $3(2-3)$ & $50(24.63)$ \\
\hline Regular meals in a day ${ }^{\mathrm{a}}$ & $1.25 \pm 0.50$ & 1.18 to 1.32 & $1(1-1)$ & $159(78.32)$ \\
\hline Food item between meals ${ }^{\mathrm{b}}$ & $2.53 \pm 0.81$ & 2.41 to 2.64 & $3(2-3)$ & $41(20.19)$ \\
\hline Daily water intake $\mathrm{c}^{\mathrm{C}}$ & $1.06 \pm 0.23$ & 1.03 to 1.09 & $1(1-1)$ & $191(94.08)$ \\
\hline Carbohydrate content (Based on glycemic-index $=\mathrm{GI})^{\mathrm{d}}$ & $1.71 \pm 0.62$ & 1.62 to 1.80 & $2(1-2)$ & $77(37.93)$ \\
\hline Vegetables $^{\mathrm{e}}$ & $1.06 \pm 0.25$ & 1.02 to 1.09 & $1(1-1)$ & $192(94.58)$ \\
\hline Fruits ${ }^{\mathrm{e}}$ & $2.65 \pm 0.71$ & 2.55 to 2.74 & $3(3-3)$ & $29(14.28)$ \\
\hline Cake, sweet biscuit, Sweets and chocolate ${ }^{f}$ & $1.28 \pm 0.63$ & 1.19 to 1.36 & $1(1-1)$ & $167(82.26)$ \\
\hline Sugary drinks ${ }^{\mathrm{f}}$ & $1.28 \pm 0.59$ & 1.19 to 1.36 & $1(1-1)$ & $162(79.08)$ \\
\hline Butter, fat, ghee, etc. ${ }^{f}$ & $1.33 \pm 0.67$ & 1.24 to 1.43 & $1(1-1)$ & $159(78.32)$ \\
\hline Soybean, egg, full-fat cheese, processed meat ${ }^{\mathrm{i}}$ & $1.67 \pm 0.80$ & 1.56 to 1.78 & $1(1-2)$ & $110(54.18)$ \\
\hline Savory foods ${ }^{i}$ & $1.70 \pm 0.86$ & 1.58 to 1.82 & $1(1-3)$ & $115(56.65)$ \\
\hline Fast foods ${ }^{\mathrm{i}}$ & $1.13 \pm 0.46$ & 1.06 to 1.19 & $1(1-1)$ & $187(92.11)$ \\
\hline Alcohol $^{\mathrm{i}}$ & $1.02 \pm 0.17$ & 1.00 to 1.04 & $1(1-1)$ & 200(98.52) \\
\hline Fish $^{g}$ & $2.44 \pm 0.73$ & 2.34 to 2.54 & $3(2-3)$ & $29(14.28)$ \\
\hline Milk $^{\mathrm{h}}$ & $1.48 \pm 0.70$ & 1.39 to 1.58 & $1(1-2)$ & $130(64.03)$ \\
\hline
\end{tabular}

$\mathrm{SD}=$ standard deviation, $\mathrm{IQR}=$ inter quartile range, $\mathrm{CI}=$ confidence interval.

a 'Never, very rarely or less than once a week $=3$ ' ' $1-4$ times a week $=2$ ' ' 5 times to every day in a week $=1$ '.

b '5 times to everyday in a week $=3$ ' ' $1-4$ times a week $=2$ ' 'Never, very rarely or less than once a week $=1$ '.

c 'Less than $1 \mathrm{~L}$ everyday' ' $1-2.5 \mathrm{~L}$ everyday ' '2.5-4 L everyday.

d 'High glycemic index items' 'medium glycemic index items' 'low glycemic index items'.

e 'Never, very rarely or less than once a week $=3$ ' ' $2-6$ times a week $=2$ ' ' $1-3$ or more times every day in a week $=1$ '.

f ' $1-3$ or more times every day in a week $=3$ ' ' $2-6$ times a week $=2$ 'Never, very rarely or less than once a week $=1$ '.

$\mathrm{g}$ 'Never $=3$ ' 'less than once a week $=2$ ' ' Once, twice or more times per week'.

$\mathrm{h}$ 'Full fat milk = 3' 'Semi-skimmed or mixed milk =2' 'None, non dairy or skimmed milk = 1 .

i 'Nearly daily, twice or more times per day $=3$ ' ' $1-5$ times a week $=2$ ' ' 1 'Never, very rarely or less than once a week = 1 '.

obesity. Although PP2 and HbA1c have not shown significant difference between both sexes. Female group had a higher level of FBG ( $\mathrm{p}<0.054)$, TC ( $\mathrm{p}<0.003$ ), TG, HDL, LDL and non-HDL cholesterol whereas urea and Creatinine concentrations were non significantly higher in male group. There were no significant difference of zinc, vitamin D, albumin and calcium between both group.

Table 3 represents the dietary assessment by utilizing the derivative questionnaire of UK Diabetes and Diet Questionnaire. The mean values of diet scores ranged from $1.02 \pm 0.17(\mathrm{CI}=1.00$ to 1.04$)$ for Alcohol to $2.65 \pm 0.71(\mathrm{CI}=2.55$ to 2.74$)$ for Fruits considered as healthiest and least healthy dietary choices respectively. $98 \%$ of patients were not consuming alcohol, reported as healthiest dietary choice while only $14 \%$ of patients were reported to take appropriate fruit content, which indicates the least healthy dietary choice. The $85 \%$ of patients were in the habit of eating Fish which is reported to be unhealthy with the mean score of $2.44 \pm 0.73(\mathrm{CI}=2.34$ to 2.54 ). Only $24.63 \%$ patients showed a good habit of taking breakfast within $2 \mathrm{~h}$ after waking. $20 \%$ patients were avoiding the unnecessary food items between the meals. The intermediate glycemic index carbohydrate foods were consumed by $37.93 \%$ of included patients. The other dietary choices were found healthy in the majority of patients. The evaluation of other factors like weight concern, physical exercise, the importance of diet change and patient confidence to change dietary habit are depicted in Table 4.
Majority of patients (73.89\%) were not concerned about their weight. Approximately $38.91 \%$ patients were doing the day to day activities. While interviewing $48 \%$ of patients said that it is a little bit important for them to change the diet and $52 \%$ of patients were moderately confident to change their diet.

The overall relationship between quintiles of the diet-quality score and anthropometric, biochemical and nutritional parameters of the study population is summarized in Table 5. No significant difference was observed between the three quintiles for age $(p=0.295)$ and total cholesterol $(p=0.450)$. However, the other parameters were found to be significant. The most striking differences were observed between the quintiles for PP2 ( $p=0.001)$, FBG $(p=0.003)$ and HbA1c $(p=0.003)$ followed by BMI $(p=0.008)$. In addition, significant differences were also found for vitamin $\mathrm{D}(\mathrm{p}=0.029)$, zinc $(\mathrm{p}=0.033)$, calcium ( $\mathrm{p}=0.044)$ and albumin $(\mathrm{p}=0.046)$. The BMI, HbA1c, FBG, PP2 and Calcium levels were directly increasing with increments in quintile values as from Q1 to Q5 whereas, zinc, vitamin D and albumin concentrations were found to be decreasing with increase in total dietary scores.

Table 6 represents the correlation of the total dietary score with BMI and other biochemical and nutritional parameters. A positive correlation was observed with BMI $(r=0.139)$, HbA1c $(r=0.253)$, FBG $(r=0.183)$, PP2 $(r=0.233)$, total cholesterol $(r=0.085)$ and calcium

Table 4

Assessment of other factors based on the derivative of UK Diabetes and Diet Questionnaire (UKDDQ).

\begin{tabular}{|c|c|c|c|c|}
\hline Other Factors $(\mathrm{N}=203)$ & Mean \pm SD & $95 \% \mathrm{CI}$ & Median(IQR) & $\mathrm{N}(\%)$ of patients w.r.t median \\
\hline Concerned about weight $^{\mathrm{a}}$ & $1.38 \pm 0.73$ & 1.28 to 1.49 & $1(1-2)$ & $150(73.89)$ \\
\hline Daily exercising ${ }^{\mathrm{b}}$ & $2.64 \pm 0.88$ & 2.51 to 2.76 & $3(2-3)$ & $79(38.91)$ \\
\hline Important to change diet ${ }^{\mathrm{c}}$ & $2.57 \pm 0.71$ & 2.47 to 2.67 & $2(2-3)$ & $99(48.76)$ \\
\hline Confident about diet change ${ }^{\mathrm{d}}$ & $2.01 \pm 0.78$ & 1.91 to 2.12 & $2(1.5-2)$ & $107(52.70)$ \\
\hline
\end{tabular}

$\mathrm{SD}=$ standard deviation, IQR = inter quartile range, $\mathrm{CI}=$ confidence interval.

a 'I am very much concerned about my weight $=4$ ' 'I am moderately concerned about my weight $=3$ ' 'I am a little concerned about my weight $=2$ ' 'I am not concerned about my weight $=1$ '.

b 'Never exercising $=4$ ' 'day to day activities $=3$ ' 'moderately exercising $=2$ ' 'as per recommended $=1$ '.

c 'Extremely important $=4$ ' 'moderately important $=3$ ' 'little bit important $=2$ ' 'Not at all $=1$ '.

d ${ }^{\prime}$ Not at all $=4$ ' 'little bit confident $=3$ ' 'moderately confident $=2$ ' 'Extremely confident $=1$ '. 
Table 5

The overall relationship between quintiles of diet-quality score and anthropometric, biochemical and nutritional parameters of study population.

\begin{tabular}{|c|c|c|c|c|c|}
\hline Variables & All patients Mean \pm SD $(n=203)$ & Q1 Mean $\pm S D(n=44)$ & Q3 Mean \pm SD $n=69$ & Q5Mean \pm SD $n=90$ & $\mathrm{P}$ \\
\hline Age & $53.92 \pm 10.56$ & $55.57 \pm 11.13$ & $54.46 \pm 10.98$ & $52.70 \pm 9.91$ & 0.295 \\
\hline BMI & $25.75 \pm 3.46$ & $24.71 \pm 2.70$ & $25.39 \pm 2.85$ & $26.55 \pm 4.03$ & 0.008 \\
\hline Weight & $62.54 \pm 10.33$ & $61.32 \pm 9.61$ & $63.26 \pm 11.10$ & $62.54 \pm 10.33$ & 0.623 \\
\hline Height & $154.63 \pm 7.93$ & $155.77 \pm 7.58$ & $153.64 \pm 8.35$ & $154.82 \pm 7.75$ & 0.361 \\
\hline W.C & $90.09 \pm 7.69$ & $88.08 \pm 6.14$ & $89.50 \pm 6.90$ & $91.54 \pm 8.68$ & 0.037 \\
\hline HbA1c & $8.28 \pm 2.34$ & $7.71 \pm 1.87$ & $7.84 \pm 2.19$ & $8.90 \pm 2.52$ & 0.003 \\
\hline FBG & $168.20 \pm 74.52$ & $148.16 \pm 60.11$ & $155.81 \pm 57.02$ & $187.49 \pm 87.64$ & 0.003 \\
\hline PP2 & $249.96 \pm 106.53$ & $210.64 \pm 80.38$ & $238.59 \pm 100.24$ & $277.90 \pm 115.35$ & 0.001 \\
\hline WHtR & $0.58 \pm 0.96$ & $0.56 \pm 0.81$ & $0.58 \pm 0.82$ & $0.59 \pm 1.12$ & 0.002 \\
\hline T. C. & $169.94 \pm 39.53$ & $164.36 \pm 32.31$ & $168.99 \pm 46.52$ & $173.40 \pm 36.89$ & 0.450 \\
\hline Zinc & $54.19 \pm 10.70$ & $57.49 \pm 15.27$ & $54.44 \pm 8.46$ & $52.38 \pm 9.16$ & 0.033 \\
\hline Vitamin D & $26.13 \pm 6.88$ & $28.18 \pm 7.46$ & $26.45 \pm 7.62$ & $24.88 \pm 5.68$ & 0.029 \\
\hline Calcium & $9.62 \pm 1.79$ & $9.04 \pm 1.18$ & $9.70 \pm 1.68$ & $9.85 \pm 2.06$ & 0.044 \\
\hline Albumin & $3.64 \pm 0.41$ & $3.78 \pm 0.50$ & $3.61 \pm 0.35$ & $3.60 \pm 0.39$ & 0.046 \\
\hline
\end{tabular}

Abbreviations: $\mathrm{SD}=$ standard deviation, $\mathrm{BMI}=$ body mass index, HbA1c $=$ glycated haemoglobin, $\mathrm{FBG}=$ fasting blood glucose, $\mathrm{PP} 2=2 \mathrm{~h}$ after meal glucose T.C $=$ total cholesterol.

$(\mathrm{r}=0.106)$ whereas, dietary score showed a negative correlation with zinc $(r=-0.116)$, vitamin $D(r=-0.185)$ and albumin $(r=-0.043)$. Furthermore, the most significant positive correlation was observed with HbA1c ( $\mathrm{p}=<0.001)$, PP2 ( $<0.001)$ and FBG $(\mathrm{p}=0.009)$, whereas negative correlation was observed with Vitamin D $(\mathrm{p}=0.008)$.

\section{Discussion}

The present study investigates the association between dietary patterns, cardio-metabolic risk and status of nutritional biomarkers in T2DM in the eastern state of Indian population using a validated measure of derivative of UKDDQ, a new dietary assessment tool. Dietary scores were categorized to be healthy at lower scores $[21.86 \pm 1.23(\mathrm{Q} 1)]$ and unhealthy at higher scores $(28.94 \pm 2.24$ (Q5)). We found higher scores mainly in patients who were living with habit of skipping breakfast. It was predominantly found in those housewives who routinely work in morning for late hours in the kitchen or some other home managing activities. ${ }^{29}$ The majority of patients were not restricted to a timely or regular meal habits. Instead, they showed a habit of eating food items extra to their required normal meals. Most of the respondents who were not getting proper fruit and fish content were seen to have a higher risk of diabetes which was in consonance with the study reported by van Dam RM et al. ${ }^{30}$ It has been found that the low economic status of patients and the confusion about dietary recommendations i.e. either to take or not to take fruit and fish was the reason behind such unhealthy nature of fruit and fish diet. A higher percentage $(62 \%)$ of patients showed a habit of taking high to moderate glycemic index items i.e. as parboiled rice, wheat parantha, noodles, potato, green pea etc. which are supposed to have a higher risk of type 2 diabetes. These results supported the existing evidence from study of Bhupathiraju Shilpa N et al. who have evaluated the T2DM risk with of food patterns based on glycemic index. ${ }^{31}$ A considerable number as $35 \%$ of patients were taking full-fat milk which was reported to be a pathogenic factor for diabetes by Schrezenmeir J. ${ }^{32}$

It was observed, $48 \%$ of patients were economically from lower background as a result having a less sedentary lifestyles. The study reported lower dietary scores for other food habits i.e. taking of fast foods (burgers, pizza, fried non-veg. or curries) and sugary soft drinks (non-diet fizzy drinks, squashes, sweetened and flavored drinks), oily or buttery items, savoury foods and other sweet chocolate, cake or biscuit items. This less level of sedentary lifestyle was also reported by Rena R. Wing et al. as healthier habit of eating through their review. ${ }^{33}$ Due to recent complete alcohol ban in Bihar, a very interesting fact was found from study results, as a negligible percentage of only $1.5 \%$ of study participants were consuming alcohol which could be considered as an important milestone to increase the effectiveness of anti-diabetic treatment therapy. This fact also supports the analysis done by Debabrata Samanta and Shivani Narayan who have analyzed recently the human development in Bihar after the alcohol prohibition. ${ }^{34}$

In an attempt to assess the relationship of different variables, a significant positive association of dietary scores with BMI, HbA1c, FBG, PP2, total cholesterol and calcium whereas a negative relationship was found with vitamin D, zinc and Albumin. Present study outcomes were not surprising as multiple previous study data has already shown the increased risk of metabolic disorders like obesity and diabetes with above discussed food habit. ${ }^{35-37}$ But the novel idea of this study is the assessment of dietary score association with other biochemical and nutritional biomarkers which is being considered as important predictors of T2DM status. The results of this study are in agreement with the study reported from seven European Union Countries by Rosalind Fallaize et al. which has also analyzed the association of diet quality scores with adiposity, total cholesterol and other nutritional biomarkers in the adult population. ${ }^{25}$

The study has also evaluated the role of diet in disease outcomes by comparing the different dietary score quintile groups. The differences between the quintile groups were found significant $(p<0.05)$. Most significant differences were seen between PP2, FBG, HbA1c, BMI followed by Zinc, vitamin D and Albumin. Increase in scores i.e. moving from lower quintile Q1 to higher score quintile Q5, the parameters like PP2 then FBG, HbA1c and BMI showed a direct relationship whereas, Zinc, vitamin D and Albumin were inversely correlated. Similar findings were also shown by Schröder $\mathrm{H}$ et al. from Spain, reporting that increased BMI is associated with impaired glucose metabolism and

Table 6

Correlation between total dietary scores and BMI, biochemical and nutritional parameters of diabetic patients.

\begin{tabular}{|c|c|c|c|c|c|c|c|c|c|}
\hline Total Dietary score & BMI & HbA1c & FBG & PP2 & T.C. & Zinc & Vitamin D & Calcium & Albumin \\
\hline Pearson ' $r$ ' & 0.139 & 0.253 & 0.183 & 0.233 & 0.085 & -0.116 & -0.185 & 0.106 & -0.043 \\
\hline $\mathrm{P}$ & 0.049 & $<0.001$ & 0.009 & 0.001 & 0.227 & 0.099 & 0.008 & 0.131 & 0.545 \\
\hline
\end{tabular}

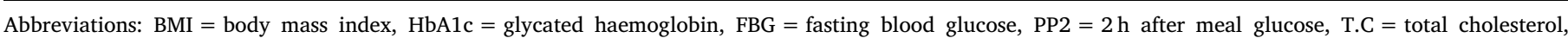
$\mathrm{r}=$ Pearson correlation coefficient. 
development of diabetes. ${ }^{38}$ Furthermore, this study extends the literature for already known research which has shown the nutritional biomarker deficiencies for vitamin D, zinc, albumin and intracellular calcium had an established function in diabetes pathophysiology. ${ }^{14-16}$

Food patterns are adopted on the basis of a number of factors like knowledge of dietary suggestions, agricultural production, social, religious, cultural and traditional identities. Due to a huge diversity of these factors, the evaluation of eating patterns among the Indian diabetics becomes a complex process. ${ }^{39-41}$ While planning nutritional therapy interventions, focus on dietary patterns with consideration of nutrient status can be a major tool to implement in diabetes management programs. Bihar has a mostly rural population with low GDP and literacy rate in India, ${ }^{5}$ so it is of great importance to improve adherence towards food pattern recommendations by providing proper nutritional education and counseling to patients which can result to better therapeutic outcomes and a reduction in overall disease burden.

Several strengths and limitations can be counted in the study. Strengths include firstly, the study was done from an economically less grown state of India where the health services are not meeting the priority needs of the population. Also, the research on diabetes is at its bottom and the people are in need of necessary valuable dietary advices. Secondly, the samples were selected randomly and we used a derivative of a new, validated diet assessment questionnaire. Limitations of the study can be counted as firstly, we used the derivative questionnaire after keeping the measuring scales same on a small sample size. Also for this cross-sectional study the covariate measurements were crudely done after adjusting the different lifestyle factors. Secondly, a short list of dietary patterns was included.

\section{Conclusion}

In conclusion, the present study shown a variable association for different parameters. Adiposity, blood glucose parameters, cholesterol and serum calcium were positively associated with dietary scores whereas Zinc, vitamin D and Albumin were negatively related. Increasing dietary scores show a provoking effect on disease condition ultimately giving worse outcomes at higher scores. The findings from nutritional biomarkers suggested that deficiencies found in specified nutrients must be supplemented as a part of treatment therapy. All the patients should be counselled, educated and encouraged regarding food habit patterns to show a greater adherence toward nutritional and medication therapy. Furthermore, a real world studies are required to robust the present findings.

\section{Ethics statement}

The study protocol was approved by the Institutional Ethics Committee of Rajendra Memorial Research Institute of Medical Sciences (RMRIMS), Patna (Reference No.41/RMRI/EC/2017).

\section{Funding}

This research received no specific grant from any funding agency, commercial or not-for-profit sectors.

\section{Conflicts of interest} tion.

There is no known conflict of interest associated with this publica-

\section{Authors contribution}

Conceived and designed the experiments: $\mathrm{AB}, \mathrm{KM}, \mathrm{KP}$.

Performed the experiments: AB, AK, CSL.

Analyzed the data: $\mathrm{AB}, \mathrm{AK}$, NAS.

Wrote the paper: AB, AK, MA, KM, IR, KP.

\section{Acknowledgments}

Authors would like to thank laboratory staff of central diagnostic laboratory, RMRIMS-Patna who helped in all the laboratory measurements. We also thank Clare Y England(clare.england@bristol.ac.uk) for allowing the use of derivative questionnaire of UK Diet and Diabetes Questionnaire (UKDDQ).

\section{Appendix A. Supplementary data}

Supplementary data to this article can be found online at https:// doi.org/10.1016/j.cegh.2019.04.007.

\section{References}

1. Inzucchi SE, Sherwin RS. Type 2 Diabetes Mellitus. Cecil Medicine. 24th ed. Philadelphia, Pa: Saunders Elsevier; 2011.

2. World Health Organization. Definition, diagnosis and classification of diabetes mellitus and its complications: report of a WHO consultation. Part 1, Diagnosis and classification of diabetes mellitus.

3. Dominguez LJ, Bes-Rastrollo M, Basterra-Gortari FJ, Gea A, Barbagallo M, MartínezGonzález MA. Association of a dietary score with incident type 2 diabetes: the Dietary-Based Diabetes-Risk Score (DDS). PLoS One. 2015 Nov 6;10(11):e0141760.

4. International Diabetes Federation. IDF Diabetes Atlas. eighth ed. 2017; 2017 Available at: http://www.idf.org/diabetesatlas.

5. Anjana RM, Deepa M, Pradeepa R, et al. Prevalence of diabetes and prediabetes in 15 states of India: results from the ICMR-INDIAB population-based cross-sectional study. Lancet Diabetes Endocrinol. 2017 Aug 1;5(8):585-596.

6. Shridhar K, Ambika Satija A, Dhillon PK, et al. Association between empirically derived dietary patterns with blood lipids, fasting blood glucose and blood pressure in adults- the India migration study. Nutr J. 2018;17:15.

7. Malik VS, Popkin BM, Bray GA, Després JP, Willett WC, Hu FB. Sugar-sweetened beverages and risk of metabolic syndrome and type 2 diabetes: a meta-analysis. Diabetes Care. 2010 Nov 1;33(11):2477-2483.

8. Mozaffarian D. Dietary and policy priorities for cardiovascular disease, diabetes, and obesity: a comprehensive review. Circulation. 2016 Jan 12;133(2):187-225.

9. Whincup PH, Donin AS. Cereal fibre and type 2 diabetes: time now for randomised controlled trials? Diabetologia. 2015 Jul 1;58(7):1383-1385

10. Korat AV, Willett WC, Hu FB. Diet, lifestyle, and genetic risk factors for type 2 diabetes: a review from the nurses' health study, nurses' health study 2, and health professionals' follow-up study. Curr Nutr Rep. 2014 Dec 1;3(4):345-354.

11. Ley SH, Hamdy O, Mohan V, Hu FB. Prevention and management of type 2 diabetes: dietary components and nutritional strategies. Lancet. 2014 Jun 7;383(9933):1999-2007.

12. Emadian A, England CY, Thompson JL. Dietary intake and factors influencing eating behaviours in overweight and obese South Asian men living in the UK: mixed method study. BMJ open. 2017 Jul 1;7(7):e016919.

13. Cross-Bardell L, George T, Bhoday M, Tuomainen H, Qureshi N, Kai J. Perspectives on enhancing physical activity and diet for health promotion among at-risk urban UK South Asian communities: a qualitative study. BMJ open. 2015 Feb 1;5(2):e007317.

14. Pittas AG, Lau J, Hu FB, Dawson-Hughes B. The role of vitamin D and calcium in type 2 diabetes. A systematic review and meta-analysis. J Clin Endocrinol Metab. 2007 Jun 1;92(6):2017-2029.

15. Jansen J, Karges W, Rink L. Zinc and diabetes_clinical links and molecular mechanisms. J Nutr Biochem. 2009 Jun 1;20(6):399-417.

16. Dolhofer R, Wieland $\mathrm{OH}$. Increased glycosylation of serum albumin in diabetes mellitus. Diabetes. 1980 Jun 1;29(6):417-422.

17. Hu FB. Dietary pattern analysis: a new direction in nutritional epidemiology. Curr Opin Lipidol. 2002 Feb 1;13(1):3-9.

18. Green R, Milner J, Joy EJ, Agrawal S, Dangour AD. Dietary patterns in India: a systematic review. Br J Nutr. 2016 Jul;116(1):142-148 20-19. Cheung BM. The cardiovascular continuum in Asia-a new paradigm for the metabolic syndrome.

19. National Research Council. Diet and Health: Implications for Reducing Chronic Disease Risk. National Academies Press; 1989 Jan 15.

20. Green R, Milner J, Joy EJ, Agrawal S, Dangour AD. Dietary patterns in India: a systematic review. Br J Nutr. 2016;116(1):142-148.

21. General Assembly of the World Medical Association. World Medical Association Declaration of Helsinki: ethical principles for medical research involving human subjects. J Am Coll Dent. 2014;81(3):14.

22. Von Elm E, Altman DG, Egger M, et al. The Strengthening the Reporting of Observational Studies in Epidemiology (STROBE) statement: guidelines for reporting observational studies. PLoS Med. 2007 Oct 16;4(10):e296.

23. Hulley SB, Newman TB, Cummings SR. Planning the measurements: precision, accuracy, and validity. Designing Clinical Research. fourth ed. Philadelphia, PA: Wolters Kluwer Health/Lippincott Williams \& Wilkins; 2013:32-42.

24. England CY, Thompson JL, Jago R, Cooper AR, Andrews RC. Development of a brief, reliable and valid diet assessment tool for impaired glucose tolerance and diabetes: the UK diabetes and diet questionnaire. Publ Health Nutr. 2017 Feb;20(2):191-199.

25. Fallaize R, Livingstone KM, Celis-Morales C, et al. Association between diet-quality scores, adiposity, total cholesterol and markers of nutritional status in european adults: findings from the Food4Me study. Nutrients. 2018 Jan 6;10(1):49. 
26. American Diabetes Association. Diagnosis and classification of diabetes mellitus. Diabetes Care. 2013 Jan;36(Suppl 1):S67.

27. Sterne JA, Smith GD. Sifting the evidence-what's wrong with significance tests? Phys Ther. 2001 Aug 1;81(8):1464-1469.

28. Yadav D, Mishra M, Tiwari A, Bisen PS, Goswamy HM, Prasad GB. Prevalence of dyslipidemia and hypertension in Indian type 2 diabetic patients with metabolic syndrome and its clinical significance. Osong Public Health Res Perspect. 2014 Jun 1;5(3):169-175.

29. Reutrakul S, Hood MM, Crowley SJ, Morgan MK, Teodori M, Knutson KL. The relationship between breakfast skipping, chronotype, and glycemic control in type 2 diabetes. Chronobiol Int. 2014 Feb 1;31(1):64-71.

30. van Dam RM, Rimm EB, Willett WC, Stampfer MJ, Hu FB. Dietary patterns and risk for type 2 diabetes mellitus in US men. Ann Intern Med. 2002 Feb 5;136(3):201-209.

31. Bhupathiraju SN, Tobias DK, Malik VS, et al. Glycemic index, glycemic load, and risk of type 2 diabetes: results from 3 large US cohorts and an updated meta-analysis. Am J Clin Nutr. 2014 Apr 30;100(1):218-232.

32. Schrezenmeir J. Hyperinsulinemia, hyperproinsulinemia, and insulin resistance in the metabolic syndrome. Experientia. 1996;5:426-432.

33. Wing RR, Goldstein MG, Acton KJ, et al. Behavioral science research in diabetes: lifestyle changes related to obesity, eating behavior, and physical activity. Diabetes Care. 2001 Jan 1;24(1):117-123.
34. Samanta D, Narayan S. Human development in Bihar and impact of liquor prohibition policy: an analysis. J Gov Public Policy. 2017 Jul 1;7(2).

35. Sahyoun NR, Jacques PF, Zhang XL, Juan W, McKeown NM. Whole-grain intake is inversely associated with the metabolic syndrome and mortality in older adults-. Am $J$ Clin Nutr. 2006 Jan 1;83(1):124-131.

36. Liu S, Song Y, Ford ES, Manson JE, Buring JE, Ridker PM. Dietary calcium, vitamin $\mathrm{D}$, and the prevalence of metabolic syndrome in middle-aged and older US women. Diabetes Care. 2005 Dec 1;28(12):2926-2932.

37. Esmaillzadeh A, Kimiagar M, Mehrabi Y, Azadbakht L, Hu FB, Willett WC. Fruit and vegetable intakes, C-reactive protein, and the metabolic syndrome-. Am J Clin Nutr. 2006 Dec 1;84(6):1489-1497.

38. Schröder H, Marrugat J, Vila J, Covas MI, Elosua R. Adherence to the traditional Mediterranean diet is inversely associated with body mass index and obesity in a Spanish population. J Nutr. 2004 Dec 1;134(12):3355-3361.

39. Atlas D. International Diabetes Federation. IDF Diabetes Atlas. seventh ed. Brussels, Belgium: International Diabetes Federation; 2015.

40. Vecchio MG, Paramesh EC, Paramesh H, et al. Types of food and nutrient intake in India: a literature review. Indian J Pediatr. 2014 Sep 1;81(1):17-22.

41. Satija A, Hu FB, Bowen L, et al. Dietary patterns in India and their association with obesity and central obesity. Publ Health Nutr. 2015;18(16):3031-3041. 